\title{
Improved First-Principles Calculation of the Third Virial Coefficient of Helium
}

\section{Giovanni Garberoglio}

Interdisciplinary Laboratory for Computational Science (LISC), FBK-CMM and University of Trento,

Via Sommarive 18, I-38123 Povo, Italy

\section{Michael R. Moldover}

Temperature, Pressure, and Flow Metrology Division, National Institute of Standards and Technology,

Gaithersburg, MD 20899

\section{Allan H. Harvey}

Thermophysical Properties

Division, National Institute of Standards and Technology, Boulder, CO 80305

garberoglio@fbk.eu michael.moldover@nist.gov allan.harvey@nist.gov

\begin{abstract}
We employ state-of-the-art pair and three-body potentials with path-integral Monte Carlo (PIMC) methods to calculate the third density virial coefficient $C(T)$ for helium. The uncertainties are much smaller than those of the best experimental results, and approximately one-fourth the uncertainty of our previous work. We have extended our results in temperature down to $2.6 \mathrm{~K}$, incorporating the effect of spin statistics that become important below approximately $7 \mathrm{~K}$. Results are given for both the ${ }^{3} \mathrm{He}$ and ${ }^{4} \mathrm{He}$ isotopes. We have also performed PIMC calculations of the third acoustic virial coefficient $\gamma_{a}$; our calculated values compare well with the limited experimental data available. A correlating equation for $C(T)$ of ${ }^{4} \mathrm{He}$ is presented; differentiation of this equation provides a reliable and simpler way of calculating $\gamma_{\mathrm{a}}$.
\end{abstract}

Key words: acoustic virial coefficients; calibration; density; helium; metrology; thermodynamic properties; virial coefficients.

Accepted: June 21, 2011

\section{Introduction}

Accurate knowledge of the thermophysical properties of helium is desirable for many applications in metrology [1-3]. At low and moderate densities, thermodynamic properties are well described by the virial expansion, which gives a rigorous series of corrections to ideal-gas behavior:

$$
\frac{p}{\rho R T}=1+B(T) \rho+C(T) \rho^{2}+\ldots
$$

In Eq. (1), $p$ is the pressure, $\rho$ the molar density, $R$ the molar gas constant, and $T$ the absolute temperature. The second virial coefficient $B(T)$ depends only on interactions between pairs of molecules, while the third virial coefficient $C(T)$ depends on interactions among three molecules.

In 2009, two of us reported [4] path-integral Monte Carlo (PIMC) calculations of the third virial coefficient $C(T)$ for ${ }^{4} \mathrm{He}$ at temperatures from $24.5661 \mathrm{~K}$ (corresponding to the triple point of neon) to $10000 \mathrm{~K}$. The values of $C(T)$ reported in Ref. [4] were based on a 
representation of the pair potential [5] and a three-body potential [6] that were state-of-the-art (or nearly so) at the time the work was performed.

The uncertainties obtained in Ref. [4] were smaller than those of the best experimental results by approximately an order of magnitude, with the majority of the uncertainty coming from the three-body potential. Further improvement is desirable - for example, in a proposed pressure standard based on capacitance measurement at $273.16 \mathrm{~K}$, the uncertainty in $C$ is still the largest contributor to the uncertainty budget between approximately $8 \mathrm{MPa}$ and $20 \mathrm{MPa}$ [7]. It is also desirable to extend the results to lower temperatures, where helium plays an important role in temperature metrology.

Recently, a state-of-the-art pair potential for helium has been developed $[8,9]$. The new potential incorporates not only extremely accurate results for the potential energy in the Born-Oppenheimer (BO) approximation [10], but also accurate calculations for the most important post-BO effects (adiabatic, relativistic, and quantum electrodynamics). The claimed uncertainty of the new pair potential is at least one order of magnitude smaller than that of the potential we used in Ref. [4]. This pair potential has been used to obtain highly accurate values for the second virial coefficient $B(T)$ and for the low-density limits of the viscosity and thermal conductivity [9]. In addition, a new three-body potential has been developed at the full-configurationinteraction (FCI) level [11], reducing the uncertainty of the three-body potential by approximately a factor of five compared to that used in Ref. [4].

In this work, we take advantage of the availability of these better potentials, and of increased computing capabilities and algorithmic improvements, to recalculate $C(T)$ with lower uncertainty than could be obtained in Ref. [4] and to extend our calculations to lower temperatures. We also extend our work to the third acoustic virial coefficient, and give some results for the ${ }^{3} \mathrm{He}$ isotope. In this paper, we will focus on aspects that differ from Ref. [4], such as the calculation of acoustic virials and the low-temperature results. The reader is referred to Ref. [4] for further background, a literature review, and details of the uncertainty analysis. Some additional details of the PIMC calculations, especially at low temperatures where spin statistics become important, are discussed in Ref. [12].

\section{Intermolecular Potentials}

For the pair potential, we use the potential first presented by Przybytek et al. [8] and described in more detail by Cencek et al. [9]. A function for the uncertainty of this potential is given in the Supplemental Material for Ref. [8], so that upper- and lower-bound potentials can be obtained by adding or subtracting the uncertainty function from the recommended pair potential. While Przybytek et al. do not attach a rigorous statistical interpretation to their "uncertainty," we believe that it is reasonable to treat it as an expanded uncertainty with coverage factor $k=2$, which corresponds to a $95 \%$ confidence limit. For calculations with ${ }^{3} \mathrm{He}$, a small adjustment (negligible in the context of this work) was made to scale the adiabatic correction to the pair potential [9] to account for the different mass.

For the nonadditive three-body potential of helium, we use the FCI potential of Cencek et al. [11]. This potential is stated to have a relative uncertainty of $2 \%$, which again we interpret as an expanded uncertainty at the $k=2$ level. For our uncertainty analysis, we construct perturbed three-body potentials FCI- (obtained by multiplying the corresponding potential by 1.02 where it is negative and by 0.98 where it is positive) and $\mathrm{FCI}+$ (multiplying by 0.98 where it is negative and by 1.02 where it is positive).

\section{Calculation Methods}

\subsection{Third Density Virial Coefficient}

It has been shown in Refs. [4] and [12] that the second virial coefficient $B(T)$ and the third virial coefficient $C(T)$ for a quantum system obeying Boltzmann statistics can be written as

$$
\begin{gathered}
B(T)=-2 \pi N_{\mathrm{A}} \int r^{2} \mathrm{~d} r\left[\mathrm{e}^{-\beta \bar{U}_{2}(r)}-1\right] \\
C(T)=4 B^{2}(T)-\frac{N_{\mathrm{A}}^{2}}{3} \int \mathrm{d} r_{1} \mathrm{~d} r_{2}\left[\mathrm{e}^{-\beta \bar{V}_{3}\left(r_{1}, r_{2}\right)}\right. \\
\left.-\mathrm{e}^{-\beta \bar{U}_{2}\left(r_{1}-r_{2} \mid\right)}-\mathrm{e}^{-\beta \bar{U}_{2}\left(r_{1} \mid\right)}-\mathrm{e}^{-\beta \bar{U}_{2}\left(r_{2} \mid\right)}+2\right],
\end{gathered}
$$


where $N_{\mathrm{A}}$ is Avogadro's constant, $\beta=1 / k_{\mathrm{B}} T$, and the effective two-body and three-body potentials $\bar{U}_{2}(r)$ and $\bar{V}_{3}\left(\boldsymbol{r}_{1}, \boldsymbol{r}_{2}\right)$ are given by the following path-integral expressions:

$$
\begin{gathered}
\exp \left(-\beta \bar{U}_{2}(r)\right)= \\
\oint D \boldsymbol{x}_{1} D \boldsymbol{x}_{2} \exp \left[-\left.\frac{1}{\hbar} \int_{0}^{\beta \hbar} \frac{m}{2}|| \frac{\mathrm{d} \boldsymbol{x}_{1}(\tau)}{\mathrm{d} \tau}\right|^{2}\right. \\
\left.\left.+\left|\frac{\mathrm{d} \boldsymbol{x}_{2}(\tau)}{\mathrm{d} \tau}\right|^{2}\right)+U_{2}\left(\left|\boldsymbol{r}+\boldsymbol{x}_{1}(\tau)-\boldsymbol{x}_{2}(\tau)\right|\right) \mathrm{d} \tau\right] \\
\exp \left[-\beta \bar{V}_{3}\left(\boldsymbol{r}_{1}, \boldsymbol{r}_{2}\right)\right]=\oint D \boldsymbol{x}_{1} D \boldsymbol{x}_{2} D \boldsymbol{x}_{3} \exp \left[-\frac{1}{\hbar}\right. \\
\int_{0}^{\beta \hbar} \frac{m}{2}\left(\left|\frac{\mathrm{d} \boldsymbol{x}_{1}(\tau)}{\mathrm{d} \tau}\right|^{2}+\left|\frac{\mathrm{d} \boldsymbol{x}_{2}(\tau)}{\mathrm{d} \tau}\right|^{2}+\left|\frac{\mathrm{d} \boldsymbol{x}_{3}(\tau)}{\mathrm{d} \tau}\right|^{2}\right)+ \\
\left.V_{3}\left(\boldsymbol{r}_{1}+\boldsymbol{x}_{1}(\tau), \boldsymbol{r}_{2}+\boldsymbol{x}_{2}(\tau), \boldsymbol{x}_{3}(\tau)\right) \mathrm{d} \tau\right],
\end{gathered}
$$

where $U_{2}(r)$ and $V_{3}\left(\boldsymbol{r}_{1}, \boldsymbol{r}_{2}, \boldsymbol{r}_{3}\right)$ are the two-body and three-body potential energies, respectively, and $m$ is the particle mass. In Eq. (5), the position of particle 3 in the $\tau=0$ imaginary-time slice has been fixed at the origin of the coordinate system, due to the translational invariance of the integrand. The variables $\boldsymbol{r}_{1}$ and $\boldsymbol{r}_{2}$ reduce to the position of particles 1 and 2, respectively, in the classical limit, that is, when $T$ is so high that the paths $x_{k}(\tau)(k=1,2,3)$ contributing most to the path integrals shrink to a point. A similar procedure was followed for Eq. (4), where we used the translational invariance of the integrand to fix the position of particle 2 in the $\tau=0$ imaginary-time slice at the origin. The variable $\boldsymbol{r}$ in Eq. (4) denotes the position of particle 1 in the classical limit.

The three-body potential energy is given by

$$
\begin{array}{r}
V_{3}\left(\boldsymbol{r}_{1}, \boldsymbol{r}_{2}, \boldsymbol{r}_{3}\right)=U_{3}\left(\boldsymbol{r}_{1}, \boldsymbol{r}_{2}, \boldsymbol{r}_{3}\right)+U_{2}\left(\left|\boldsymbol{r}_{1}-\boldsymbol{r}_{2}\right|\right) \\
+U_{2}\left(\left|\boldsymbol{r}_{2}-\boldsymbol{r}_{3}\right|\right)+U_{2}\left(\left|\boldsymbol{r}_{1}-\boldsymbol{r}_{3}\right|\right),
\end{array}
$$

where $U_{3}\left(\boldsymbol{r}_{1}, \boldsymbol{r}_{2}, \boldsymbol{r}_{3}\right)$ denotes the non-additive part of the three-body potential energy and $U_{2}(r)$ is the pair potential. In Eqs. (4) and (5), the path integrals are performed over all closed paths that have the origin as endpoints, that is, paths $\boldsymbol{x}(\tau)$ fulfilling the conditions $\boldsymbol{x}(0)=\boldsymbol{x}(\beta \hbar)=\mathbf{0}$. The path integrals are normalized in such a way that their discretized form reads [12]

$$
\begin{gathered}
\oint D x \exp \left[-\frac{1}{\hbar} \int_{0}^{\beta \hbar} \frac{m}{2}\left|\frac{\mathrm{d} \boldsymbol{x}(\tau)}{\mathrm{d} \tau}\right|^{2} \mathrm{~d} \tau\right] \\
\equiv \lim _{P \rightarrow \infty} \int \prod_{i=2}^{P} \mathrm{~d} \boldsymbol{x}^{(i)} \Lambda^{3}\left(\frac{P^{3 / 2}}{\Lambda^{3}}\right)^{P} \\
\exp \left[-\frac{\pi P}{\Lambda^{2}} \sum_{i=1}^{P}\left|\boldsymbol{x}^{(i+1)}-\boldsymbol{x}^{(i)}\right|^{2}\right]=1,
\end{gathered}
$$

where $\boldsymbol{x}^{(P+1)}=\boldsymbol{x}^{(1)}=\mathbf{0}$ and $\Lambda=h / \sqrt{2 \pi m k_{\mathrm{B}} T}$ is the de Broglie wavelength of a particle with mass $m$. The integrand of the middle expression in Eq. (7) can be interpreted as the probability of having a ring polymer with $P$ beads in the positions given by the coordinates $\boldsymbol{x}^{(1)}, \ldots, \boldsymbol{x}^{(\mathrm{P})}[13]$. In the following subsection, this quantity will be denoted as $F_{\text {ring }}$.

At low temperatures where Boltzmann statistics is no longer a good approximation, the above equations must be extended to incorporate Bose-Einstein or Fermi-Dirac statistics. The details of this extension are given in Ref. [12], where it was shown that the incorporation of spin statistics is necessary for both isotopes of helium at temperatures below approximately $7 \mathrm{~K}$.

The number of beads $P$ was chosen as a function of temperature according to $P=7+(1200 \mathrm{~K}) / T$ for ${ }^{4} \mathrm{He}$ and $P=7+(1800 \mathrm{~K}) / T$ for ${ }^{3} \mathrm{He}$, with the resulting $P$ rounded to the nearest integer. Preliminary calculations showed that these choices for $P$ provide converged results (well within the statistical uncertainty of the calculation) for $C(T)$ throughout the range of temperatures spanned by the present work. The ring polymers were generated as described in Ref. [4]. As in Ref. [4], we used the VEGAS algorithm [14] for the numerical integration of Eq. (7). We averaged 256 independent calculations, each with $10^{6}$ integration points, in order to obtain the final result and its statistical uncertainty. For low temperatures where spin statistics are significant, the additional non-Boltzmann terms required were calculated as described in Ref. [12] with 128 independent integrations. 


\subsection{Third Acoustic Virial Coefficient}

The square of the speed of sound in a gas as a function of pressure on isotherms has the low-density expansion:

$$
u^{2}=\frac{\gamma_{0} R T}{M}\left(1+\frac{\beta_{\mathrm{a}} p}{R T}+\frac{\gamma_{\mathrm{a}} p^{2}}{R T}+\frac{\delta_{\mathrm{a}} p^{3}}{R T}+\cdots\right) .
$$

Here, $u$ is the speed of sound; $\beta_{\mathrm{a}}, \gamma_{\mathrm{a}}$, and $\delta_{\mathrm{a}}$ are the temperature-dependent second, third, and fourth acoustic virial coefficients; $M$ is the molar mass; and $\gamma_{0} \equiv C_{p} / C_{v}$ is the ratio of the constant-pressure heat capacity to the constant-volume heat capacity in the ideal-gas state, which is exactly $5 / 3$ for a monatomic gas. [The ideal-gas heat-capacity ratio $\gamma_{0}$ should not be confused with the acoustic virial coefficient $\gamma_{\mathrm{a}}$.] Insofar as $\gamma_{a}$ is a second-order correction for non-ideality, it is analogous to the third density virial coefficient $C$. We choose to discuss $R T \gamma_{\mathrm{a}}$ instead of $\gamma_{\mathrm{a}}$ because $R T \gamma_{\mathrm{a}}$ has both the units and the order of magnitude of the more familiar third density virial coefficient $C$. Exact thermodynamic relations connect $\gamma_{\mathrm{a}}$ to the density virial coefficients $B$ and $C$ and their first two temperature derivatives [15]. These relations are:

$$
R T \gamma_{\mathrm{a}}=L-\beta_{\mathrm{a}} B
$$

where $\beta_{\mathrm{a}}$ is related to the second density virial coefficient $B(T)$ and its temperature derivatives by

$$
\beta_{\mathrm{a}}(T)=2 B+2\left(\gamma_{0}-1\right) T \frac{\mathrm{d} B}{\mathrm{~d} T}+\frac{\left(\gamma_{0}-1\right)^{2}}{\gamma_{0}} T^{2} \frac{\mathrm{d}^{2} B}{\mathrm{~d} T^{2}},
$$

and

$$
\begin{array}{r}
\gamma_{0} L(T)=\left(\gamma_{0}-1\right) Q^{2}+\left(2 \gamma_{0}+1\right) C+ \\
\left(\gamma_{0}^{2}-1\right) T \frac{\mathrm{d} C}{\mathrm{~d} T}+\frac{\left(\gamma_{0}-1\right)^{2}}{2} T^{2} \frac{\mathrm{d}^{2} C}{\mathrm{~d} T^{2}}, \\
Q(T)=B+\left(2 \gamma_{0}-1\right) T \frac{\mathrm{d} B}{\mathrm{~d} T}+\left(\gamma_{0}-1\right) T^{2} \frac{\mathrm{d}^{2} B}{\mathrm{~d} T^{2}} .
\end{array}
$$

For PIMC calculation of the acoustic virial coefficients, it is necessary to derive path-integral expressions for the temperature derivatives of the density virial coefficients. For this purpose, we use the derivatives of Eqs. (2) and (3) with respect to $\beta=1 / k_{\mathrm{B}} T$, together with the identities

$$
\begin{aligned}
& \frac{\mathrm{d}}{\mathrm{d} T}=-\beta^{2} \frac{\mathrm{d}}{\mathrm{d} \beta} \\
& \frac{\mathrm{d}^{2}}{\mathrm{~d} T^{2}}=\beta^{3}\left(2 \frac{\mathrm{d}}{\mathrm{d} \beta}+\beta \frac{\mathrm{d}^{2}}{\mathrm{~d} \beta^{2}}\right) .
\end{aligned}
$$

In Eqs. (2) and (3), the second and third density virial coefficients are given as a sum of terms that involve the integral of products of ring-polymer probability distributions with Boltzmann factors of the interaction potential averaged along the path. Making use of the fact that $\frac{\mathrm{d} \Lambda}{\mathrm{d} \beta}=\frac{\Lambda}{2 \beta}$, the derivatives of the ring-polymer probability distribution can be written as

$$
\begin{aligned}
\frac{\mathrm{d} F_{\text {ring }}}{\mathrm{d} \beta} & =\frac{1}{\beta}\left[\frac{\pi P}{\Lambda^{2}} \sum_{i=1}^{P}\left|\boldsymbol{x}^{(i+1)}-\boldsymbol{x}^{(i)}\right|^{2}-\frac{3}{2}(P-1)\right] F_{\text {ring }} \\
& \equiv \frac{a}{\beta} F_{\text {ring }},
\end{aligned}
$$

where the last equality defines the quantity $a$. The Boltzmann factors of the potential along the paths quite generally have the form

$$
W \equiv \mathrm{e}^{-\beta \bar{U}(r)}=\exp \left[\frac{-\beta}{P} \sum_{i=1}^{P} U\left(\boldsymbol{r}+\boldsymbol{x}^{(i)} \mid\right)\right]
$$

whose $\beta$ derivative is given by

$$
\begin{aligned}
\frac{\mathrm{de}^{-\beta \bar{U}(r)}}{\mathrm{d} \beta} & =-\frac{1}{P} \sum_{i=1}^{P} U\left(\boldsymbol{r}+\boldsymbol{x}^{(i)} \mid\right) \mathrm{e}^{-\beta \bar{U}(r)} \\
& \equiv \frac{b}{\beta} W
\end{aligned}
$$

where the last equality defines the quantity $b$. 
Path-integral expressions for the temperature derivatives of $B(T)$ and $C(T)$ can then be derived with the use of Eqs. (15) and (17). For example, the first temperature derivative of $B(T)$ can be written as

$$
\begin{aligned}
& \frac{\mathrm{d} B}{\mathrm{~d} T}=-2 \pi N_{\mathrm{A}} \beta \int r^{2} \mathrm{~d} r \prod_{i=2}^{P} \mathrm{~d} \boldsymbol{x}_{1}^{(i)} \mathrm{d} \boldsymbol{x}_{2}^{(i)} \\
& F_{\text {ring }}(1) F_{\text {ring }}(2)\left[\left(a_{1}+a_{2}\right)(W-1)+b W\right],
\end{aligned}
$$

where $F_{\text {ring }}(1)$ is the probability distribution for the configurations of the first ring polymer, with a corresponding definition for the second, and $a_{1}$ and $a_{2}$ are the quantity $a$ defined in Eq. (15) for the first and second ring polymer, respectively.

In the case of $C(T)$, Eq. (18) can be modified to calculate the temperature derivatives of the terms appearing in Eq. (3), taking into account the fact that the potential in the definition of $W$ is actually a threebody potential and that three ring polymers must be considered. As a consequence, there is another distribution probability $F_{\text {ring }}(3)$ for the third particle, as well as an integration over these ring-polymer configurations (that is, an additional factor $\prod_{i=2}^{P} \mathrm{~d} \boldsymbol{x}_{3}^{(i)}$ in the integration measure). Moreover, $a_{1}+a_{2}$ must be replaced by $a_{1}+a_{2}+a_{3}$, and the integral over $\mathrm{d} r$ in Eq. (18) becomes an integration over $\mathrm{d} \boldsymbol{r}_{1} \mathrm{~d} \boldsymbol{r}_{2}$ when calculating $\mathrm{d} C / \mathrm{d} T$.

To calculate the second temperature derivatives of the virial coefficients, we use Eq. (14) together with the relations

$$
\begin{gathered}
\frac{\mathrm{d}^{2} F_{\text {ring }}}{\mathrm{d} \beta^{2}}=\frac{F_{\text {ring }}}{\beta^{2}}\left[a^{2}-2 a-\frac{3}{2}(P-1)\right] \\
\frac{\mathrm{d}^{2} W}{\mathrm{~d} \beta^{2}}=\frac{W}{\beta^{2}} b(b-1) .
\end{gathered}
$$

The final result for the second derivative of $B(T)$ is

$$
\begin{aligned}
\frac{\mathrm{d}^{2} B}{\mathrm{~d} T^{2}}=-2 \pi N_{\mathrm{A}} & \beta^{2} \int r^{2} \mathrm{~d} r \prod_{i=2}^{P} \mathrm{~d} \boldsymbol{x}_{1}^{(i)} \mathrm{d} \boldsymbol{x}_{2}^{(i)} F_{\text {ring }}(1) F_{\text {ring }}(2) \\
& {\left[\left(\left(a_{1}+a_{2}\right)^{2}-3(P-1)\right)(W-1)+\right.} \\
& \left.W\left(b^{2}+2\left(a_{1}+a_{2}\right) b+b\right)\right] .
\end{aligned}
$$

The second derivative of $C(T)$ is given by a similar expression, after performing on Eq. (21) the same substitutions described above for the first derivative, together with replacing the term $3(P-1)$ by $9(P-1) / 2$.

The temperature derivatives of $C(T)$ were used to calculate $L(T)$ according to Eq. (11). The same PIMC methodology was used as described in Sec. 3.1; the values were obtained from 256 independent calculations with $10^{6}$ integration steps each.

\section{Results}

\subsection{Third Virial Coefficients}

Table 1 shows our calculated $C(T)$ for ${ }^{4} \mathrm{He}$. In addition to all the temperatures given in Ref. [4], Table 1 includes lower temperatures (including some corresponding to fixed points on the ITS-90 temperature scale) and a few additional intermediate temperatures. Spin statistics significantly affect $C(T)$ below about $7 \mathrm{~K}$; this is discussed in detail in Ref. [12], where we describe the method of incorporating these effects and show the size of the various exchange contributions at low temperatures for both ${ }^{3} \mathrm{He}$ and ${ }^{4} \mathrm{He}$.

The low-temperature values in Table 1 differ slightly from those given in Ref. [12]; we discovered a small error in our earlier implementation of the three-body potential and the values in Table 1 supersede those in Ref. [12]. These differences are smaller than the uncertainties of the calculated $C(T)$, and the conclusions of Ref. [12] are not affected.

Table 1. Third virial coefficients $C(T)$ for ${ }^{4} \mathrm{He}$ calculated in this work and our estimates (see Sec. 4.3) of their expanded $(k=2)$ uncertainties $U(C)$

\begin{tabular}{lcc}
\hline \hline $\begin{array}{c}T \\
(\mathrm{~K})\end{array}$ & $\begin{array}{c}C \\
\left(\mathrm{~cm}^{6} \cdot \mathrm{mol}^{-2}\right)\end{array}$ & $\begin{array}{c}U(C) \\
\left(\mathrm{cm}^{6} \cdot \mathrm{mol}^{-2}\right)\end{array}$ \\
\hline 2.6 & 292. & 53. \\
2.8 & 616. & 45. \\
3 & 826. & 35. \\
3.2 & 950. & 30. \\
3.5 & 1050. & 24. \\
3.7 & 1070. & 21. \\
4.0 & 1086. & 17. \\
4.2 & 1075. & 15. \\
4.5 & 1047. & 13. \\
5 & 987. & 10. \\
6 & 854.8 & 6.2 \\
7 & 744.5 & 4.2 \\
8.5 & 620.7 & 3.2 \\
\hline
\end{tabular}


Table 1. (Continued))

\begin{tabular}{|c|c|c|}
\hline $\begin{array}{c}T \\
(\mathrm{~K})\end{array}$ & $\begin{array}{c}C \\
\left(\mathrm{~cm}^{6} \cdot \mathrm{mol}^{-2}\right)\end{array}$ & $\begin{array}{c}U(C) \\
\left(\mathrm{cm}^{6} \cdot \mathrm{mol}^{-2}\right)\end{array}$ \\
\hline 10 & 530.1 & 2.3 \\
\hline 12 & 449.9 & 1.5 \\
\hline 13.8033 & 399.8 & 1.1 \\
\hline 15 & 373.8 & 1.0 \\
\hline 17 & 341.91 & 0.82 \\
\hline 18.689 & 319.86 & 0.70 \\
\hline 20 & 307.24 & 0.64 \\
\hline 24.5561 & 273.84 & 0.47 \\
\hline 30 & 248.75 & 0.37 \\
\hline 35 & 233.56 & 0.31 \\
\hline 40 & 222.08 & 0.27 \\
\hline 50 & 205.75 & 0.21 \\
\hline 63.15 & 190.87 & 0.17 \\
\hline 75 & 180.81 & 0.15 \\
\hline 83.806 & 174.59 & 0.14 \\
\hline 100 & 164.84 & 0.12 \\
\hline 120 & 155.06 & 0.10 \\
\hline 140 & 146.846 & 0.092 \\
\hline 170 & 136.791 & 0.082 \\
\hline 200 & 128.427 & 0.074 \\
\hline 223.152 & 122.916 & 0.070 \\
\hline 235 & 120.266 & 0.068 \\
\hline 250 & 117.223 & 0.067 \\
\hline 273.16 & 112.847 & 0.064 \\
\hline 293.15 & 109.426 & 0.062 \\
\hline 302.915 & 107.822 & 0.062 \\
\hline 325 & 104.493 & 0.060 \\
\hline 350 & 100.997 & 0.058 \\
\hline 375 & 97.793 & 0.057 \\
\hline 400 & 94.837 & 0.056 \\
\hline 429.75 & 91.598 & 0.055 \\
\hline 450 & 89.552 & 0.054 \\
\hline 500 & 84.934 & 0.053 \\
\hline 550 & 80.885 & 0.051 \\
\hline 600 & 77.285 & 0.050 \\
\hline 650 & 74.039 & 0.050 \\
\hline 700 & 71.104 & 0.049 \\
\hline 750 & 68.427 & 0.048 \\
\hline 800 & 65.994 & 0.048 \\
\hline 900 & 61.661 & 0.047 \\
\hline 1000 & 57.949 & 0.047 \\
\hline 1200 & 51.857 & 0.046 \\
\hline 1400 & 47.039 & 0.045 \\
\hline 1600 & 43.114 & 0.045 \\
\hline 1800 & 39.842 & 0.044 \\
\hline 2000 & 37.063 & 0.044 \\
\hline 2500 & 31.615 & 0.043 \\
\hline 3000 & 27.610 & 0.042 \\
\hline 4000 & 22.039 & 0.041 \\
\hline 5000 & 18.317 & 0.039 \\
\hline 7500 & 12.765 & 0.036 \\
\hline 10000 & 9.664 & 0.034 \\
\hline
\end{tabular}

\subsection{Third Acoustic Virial Coefficients}

In Table 2, we show results for the third acoustic virial coefficient $\gamma_{\mathrm{a}}$. Because the quantity actually calculated by PIMC is $L$ (see Sec. 3.2 and Eqs. (9) and (11)), and because of the variation of the magnitude of $\gamma_{\mathrm{a}}$ with temperature, we tabulate the quantity $R T \gamma_{\mathrm{a}}$ (and its expanded uncertainty as discussed below). Calculation of $R T \gamma_{\mathrm{a}}$ via Eq. (9) requires values of $B$ and $\beta_{\mathrm{a}}$, which we obtain from the work of Cencek et al. [9] and which have such small uncertainties that they can be considered exact in the context of these calculations of $\gamma_{\mathrm{a}}$.

Table 2. Third acoustic virial coefficients expressed as $R T \gamma_{\mathrm{a}}$ for ${ }^{4} \mathrm{He}$ calculated by PIMC and our estimates of their expanded $(k=2)$ uncertainties $U(C)$. Also shown are values calculated from the $C(T)$ correlation Eq. (22)

\begin{tabular}{|c|c|c|c|}
\hline $\begin{array}{c}T \\
(\mathrm{~K})\end{array}$ & $\begin{array}{l}R T \gamma_{\mathrm{a}}(\mathrm{PIMC}) \\
\left(\mathrm{cm}^{6} \cdot \mathrm{mol}^{-2}\right)\end{array}$ & $\begin{array}{c}U\left(R T \gamma_{\mathrm{a}}\right) \\
\left(\mathrm{cm}^{6} \cdot \mathrm{mol}^{-2}\right)\end{array}$ & $\begin{array}{l}R T \gamma_{\mathrm{a}}(\text { Eq. } 22) \\
\left(\mathrm{cm}^{6} \cdot \mathrm{mol}^{-2}\right)\end{array}$ \\
\hline 3 & - & - & -13335 \\
\hline 4 & - & - & -5250 \\
\hline 5 & - & - & -2124 \\
\hline 6 & - & - & -681 \\
\hline 7 & - & - & 57. \\
\hline 8 & - & - & 458. \\
\hline 9 & - & - & 682. \\
\hline 10 & - & - & 807 \\
\hline 12 & - & - & 906. \\
\hline 14 & - & - & 914. \\
\hline 16 & - & - & 885. \\
\hline 18 & - & - & 843. \\
\hline 20 & 736. & 83. & 796. \\
\hline 24.5561 & 715. & 51. & 695. \\
\hline 30 & 590. & 34. & 592. \\
\hline 40 & 455. & 18. & 454. \\
\hline 50 & 366. & 12. & 360. \\
\hline 63.15 & 269.9 & 7.8 & 276.4 \\
\hline 75 & 222.9 & 6.0 & 223.9 \\
\hline 83.806 & 192.9 & 4.8 & 193.9 \\
\hline 100 & 152.7 & 3.3 & 151.9 \\
\hline 120 & 113.4 & 2.8 & 115.4 \\
\hline 140 & 88.0 & 2.2 & 89.3 \\
\hline 170 & 62.4 & 1.8 & 61.9 \\
\hline 200 & 42.8 & 1.4 & 43.1 \\
\hline 223.152 & 32.3 & 1.2 & 32.3 \\
\hline 235 & 27.8 & 1.1 & 27.6 \\
\hline 250 & 22.5 & 1.1 & 22.4 \\
\hline 273.16 & 15.82 & 0.88 & 15.61 \\
\hline 293.15 & 10.18 & 0.93 & 10.71 \\
\hline 302.915 & 8.18 & 0.79 & 8.59 \\
\hline 325 & 3.90 & 0.75 & 4.32 \\
\hline
\end{tabular}


Table 2. (Continued))

\begin{tabular}{cccc}
\hline$T$ & $\begin{array}{c}R T \gamma_{\mathrm{a}}(\mathrm{PIMC}) \\
(\mathrm{K})\end{array}$ & $\begin{array}{c}U\left(R T \gamma_{\mathrm{a}}\right) \\
\left(\mathrm{cm}^{6} \cdot \mathrm{mol}^{-2}\right)\end{array}$ & $\begin{array}{c}R T \gamma_{\mathrm{a}}(\mathrm{Eq} \cdot 2) \\
\left(\mathrm{cm}^{6} \cdot \mathrm{mol}^{-2}\right)\end{array}$ \\
\hline 350 & 0.07 & 0.73 & 0.25 \\
375 & -3.90 & 0.65 & -3.19 \\
400 & -6.03 & 0.57 & -6.12 \\
429.75 & -9.27 & 0.52 & -9.06 \\
450 & -11.39 & 0.50 & -10.79 \\
500 & -14.19 & 0.46 & -14.31 \\
550 & -17.65 & 0.40 & -17.02 \\
600 & -19.31 & 0.43 & -19.12 \\
650 & -20.90 & 0.36 & -20.79 \\
700 & -21.93 & 0.34 & -22.11 \\
750 & -22.93 & 0.32 & -23.17 \\
800 & -23.98 & 0.32 & -24.02 \\
900 & -25.27 & 0.26 & -25.26 \\
1000 & -26.28 & 0.25 & -26.07 \\
1200 & -26.99 & 0.23 & -26.87 \\
1400 & -27.13 & 0.21 & -27.07 \\
1600 & -27.13 & 0.19 & -26.96 \\
1800 & -26.77 & 0.18 & -26.68 \\
2000 & -26.40 & 0.17 & -26.30 \\
2500 & -25.24 & 0.14 & -25.19 \\
5000 & -20.27 & 0.11 & -20.25 \\
10000 & -14.58 & 0.09 & -14.72 \\
\hline & & & \\
\hline
\end{tabular}

The uncertainty in the path-integral calculation of $\gamma_{\mathrm{a}}$ becomes quite large at low temperatures, which is why PIMC values at lower temperatures are not reported in Table 2. This is due to the statistical uncertainty of the Monte Carlo integration for $L$; the convergence behavior of this integration is much worse than that for $C(T)$. The reason for this difference is not completely clear, but it may be due to the fact that the quantity $a$ that is averaged in the calculation of $\mathrm{d} C / \mathrm{d} T$ [see Eq. (15)] is the so-called thermodynamic estimator of the kinetic energy. This estimator is known to be characterized by a large variance, and therefore long computations are needed to evaluate its average value with small uncertainty [16]. Moreover, the second temperature derivative needed for calculation of the acoustic virial coefficients might add further statistical noise to the calculation.

It is also possible to calculate $\gamma_{\mathrm{a}}$ from a correlation of $C(T)$, as given in Sec. 4.4, by differentiating the correlation to produce $\mathrm{d} C / \mathrm{d} T$ and $\mathrm{d}^{2} C / \mathrm{d} T^{2}$ as required in Eq. (11). The quantities involving $B$ can again be obtained from the work of Cencek et al. [9]. Values of $R T \gamma_{\mathrm{a}}$ calculated in this manner are also shown in Table 2. They are consistent with the values calculated directly by PIMC.

\subsection{Uncertainty Analysis}

The analysis of uncertainty in $C(T)$ was similar but not identical to that described in Ref. [4]. The contributing factors are the uncertainty in the pair potential, the uncertainty in the three-body potential, and the uncertainty in the convergence of the PIMC calculation.

The standard uncertainty due to PIMC convergence was estimated as the standard deviation of the mean from the 256 independent Monte Carlo runs.

The contributions due to the uncertainties in the potentials were evaluated by calculating $C(T)$ with perturbed upper- and lower-bound versions of the potentials as described in Sec. 2. In order to avoid noise introduced by the PIMC convergence uncertainty, these calculations were performed with the semiclassical method described in Sec. 3.1 of Ref. [4], which was shown to be fairly accurate down to about $50 \mathrm{~K}$. This approach to estimating uncertainty is adequate even at lower temperatures where the semiclassical values of $C(T)$ are no longer very accurate, since the needed quantity is not $C(T)$ itself but rather the difference between $C(T)$ calculated from the upper perturbed potential and $C(T)$ calculated from the lower perturbed potential.

Below $20 \mathrm{~K}$, the semiclassical results deviate sufficiently from reality that we no longer trust them for uncertainty analysis. Instead, we observe that the uncertainty due to the potentials increases slowly and smoothly as the temperature is reduced, while the statistical uncertainty of the PIMC calculation increases more quickly. Because of these trends, the contribution of the potential uncertainty, which is our largest uncertainty component above $40 \mathrm{~K}$, is roughly $60 \%$ as large as the PIMC convergence component at $20 \mathrm{~K}$. It is reasonable to assume that this trend continues, so that the uncertainty from the potentials will be less than $60 \%$ of that from the PIMC convergence at lower temperatures. Therefore, we make the conservative estimate that the potential component of the uncertainty is $60 \%$ of that from the PIMC convergence at all temperatures below $20 \mathrm{~K}$.

The last column of Table 1 shows the resulting expanded uncertainties $U(C)$ with coverage factor $k=2$. For purposes of illustration, we summarize the uncertainty calculation for the point at $273.16 \mathrm{~K}$. The standard uncertainty of the PIMC integration is $0.0069 \mathrm{~cm}^{6} \cdot \mathrm{mol}^{-2}$. The standard uncertainty due to the uncertainty of the two-body potential is $0.0042 \mathrm{~cm}^{6} \cdot \mathrm{mol}^{-2}(1 / 4$ of the difference between $C(T)$ 
calculated semiclassically with the upper- and lower-bound pair potentials, with the three-body potential of Ref. [11] used in each case). The standard uncertainty due to the three-body potential, computed analogously with perturbed three-body potentials, is $0.0311 \mathrm{~cm}^{6} \cdot \mathrm{mol}^{-2}$. These are combined in quadrature to yield a standard uncertainty $u(C)=0.0321 \mathrm{~cm}^{6} \cdot \mathrm{mol}^{-2}$, which when multiplied by two yields an expanded uncertainty $U(C)$ of $0.064 \mathrm{~cm}^{6} \cdot \mathrm{mol}^{-2}$.

The uncertainty in the acoustic third virial coefficient, shown in Table 2, is computed analogously. In this case, the analysis is less rigorous because the perturbed potentials do not necessarily define upper and lower bounds for the temperature derivatives of $C$ that contribute to $\gamma_{\mathrm{a}}$. However, at all but the highest temperatures (above $2000 \mathrm{~K}$ ), the uncertainty in $\gamma_{\mathrm{a}}$ is dominated by the convergence uncertainty in the PIMC calculations, so the expanded uncertainties shown in Table 2 should be good estimates.

\subsection{Correlation for Results}

We correlated the results for $C(T)$ in Table 1 as a function of temperature:

$$
\frac{C}{1 \mathrm{~cm}^{6} \cdot \mathrm{mol}^{-2}}=\sum_{i=1}^{6} a_{i}\left(T^{*}\right)^{b_{i}},
$$

where $T^{*}=T /(100 \mathrm{~K})$ and the parameters $a_{i}$ and $b_{i}$ are given in Table 3. Equation (22) reproduces the values in Table 1 within tolerances smaller than their expanded uncertainties $U(C)$; the fit is much closer than $U(C)$ at high temperatures where the uncertainty is dominated by the Type B contribution from the uncertainty of the potentials. It covers the entire range from $2.6 \mathrm{~K}$ to $10000 \mathrm{~K}$, but should not be extrapolated outside this range. It may be differentiated to obtain $\mathrm{d} C / \mathrm{d} T$ and $\mathrm{d}^{2} C / \mathrm{d} T^{2}$, which can be used for calculation of acoustic virial coefficients as discussed in Sec. 4.2.

It is also convenient to have a continuous function for the expanded uncertainty $U(C)$. This will naturally be approximate, as there is significant noise in the uncertainties given in Table 1. $U(C)$ can be represented reasonably well as a function of temperature over the entire range of Table 1 by

$$
\begin{aligned}
\log _{10}\left[\frac{U(C)}{1 \mathrm{~cm}^{6} \cdot \mathrm{mol}^{-2}}\right] & =3.12-3.76 \tau \\
& +1.07 \tau^{2}-0.104 \tau^{3},
\end{aligned}
$$

where $\tau=\log _{10}(T / \mathrm{K})$.
Table 3. Coefficients for Eq. (22) for the third virial coefficient of helium

\begin{tabular}{ccc}
\hline \hline$i$ & $a_{i}$ & $b_{i}$ \\
\hline 1 & 177.98 & -0.15 \\
2 & -494.87 & -0.25 \\
3 & 849.84 & -0.50 \\
4 & -1003.30 & -0.95 \\
5 & 635.18 & -1.07 \\
6 & -0.035012 & -3.15 \\
\hline
\end{tabular}

\subsection{Comparison With Experiment for $C(T)$}

Extensive comparisons with experimental $C(T)$ data were given in Ref. [4], demonstrating that the uncertainties of calculated $C(T)$ were much smaller than those obtained from experiment. Since our new values are within the expanded uncertainties of those calculated previously, we do not repeat all the comparisons because the figures would look nearly identical to those in Ref. [4]. Instead, we limit our comparisons to the important range near room temperature and to the lowtemperature range that was not covered in Ref. [4].

In Fig. 1, our results are compared to those from the two most widely used experimental sources for $C(T)$ $[17,18]$ at temperatures from $250 \mathrm{~K}$ to $325 \mathrm{~K}$. The error bars on the experimental points represent expanded uncertainties with coverage factor $k=2$, while the expanded uncertainties on our calculated points (see Table 1) are smaller than the size of the symbols. Our calculations are fully consistent with the experimental data, but have smaller uncertainties by factors of approximately 50 .

In Fig. 2, we compare our calculated $C(T)$ with the available experimental data below $40 \mathrm{~K}$ [19-24]. Error bars drawn on the points from this work represent expanded uncertainties $U(C)$ from Table 1; they are not drawn above $5 \mathrm{~K}$ because they would be smaller than the size of the symbols. For clarity, we do not draw error bars for the experimental points; in some sources $[19,20]$ these were not reported and in the others they were usually quite large (on the order of hundreds of $\mathrm{cm}^{6} \cdot \mathrm{mol}^{-2}$ ), often extending off the scale of Fig. 2. Gaiser et al. [24] described their data obtained by dielectric-constant gas thermometry from $3.7 \mathrm{~K}$ to $36 \mathrm{~K}$ with a smooth function for $C(T)$, which we show as a dashed line on Fig. 2. From a figure in Ref. [24], it appears that their expanded $(k=2)$ uncertainties would be on the order of $20 \mathrm{~cm}^{6} \cdot \mathrm{mol}^{-2}$ over most of this range, becoming somewhat larger at the lowest temperatures. 


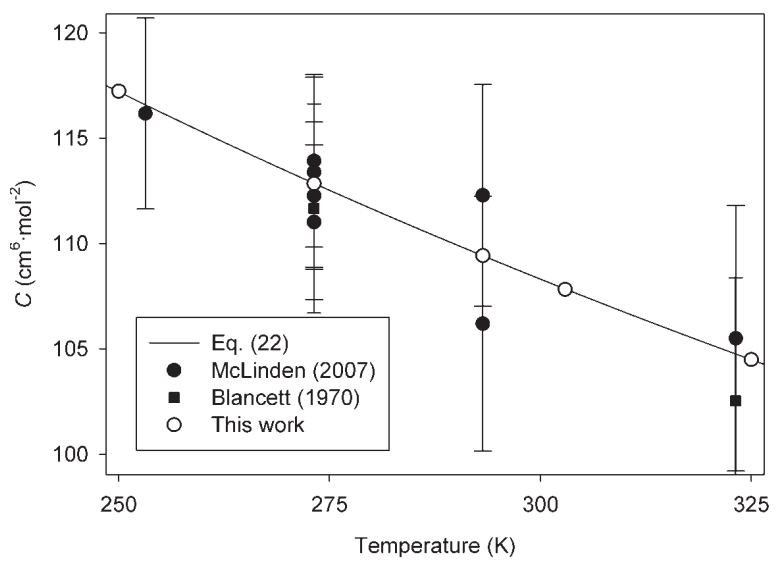

Fig. 1. Comparison of $C(T)$ for ${ }^{4} \mathrm{He}$ calculated in this work with experimental values at near-ambient temperatures. Error bars on experimental points represent expanded uncertainties with coverage factor $k=2$; uncertainties for this work (see Table 1) are not shown because the error bars would be smaller than the symbols.

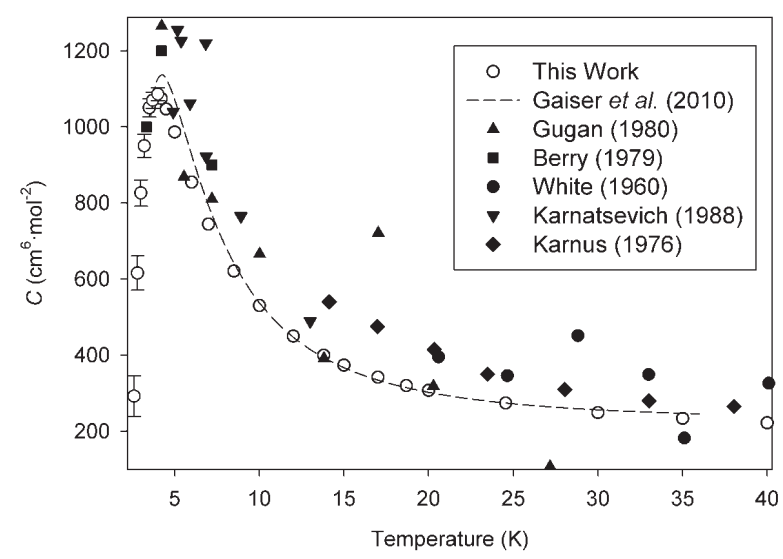

Fig. 2. Comparison of $C(T)$ for ${ }^{4} \mathrm{He}$ calculated in this work with experimental values at low temperatures. Error bars on calculated points represent expanded uncertainties with coverage factor $k=2$ (see Table 1), and are not shown when they would be smaller than the symbol size. Uncertainties for experimental points are not shown for clarity (see text).

Our results are generally consistent with the older experimental sources [19-23] within their scatter and uncertainties. We are for the most part in good agreement with the recent results of Gaiser et al. [24], with moderate disagreement at the low end of their temperature range. To examine this more closely, in Fig. 3 we plot the difference between the function of Gaiser et al. and our results as correlated by Eq. (22); Fig. 3 also shows our calculated PIMC points to demonstrate that Eq. (22) reproduces our results within their uncertainties. Our results agree with Gaiser et al. within mutual expanded uncertainties except between approximately $4 \mathrm{~K}$ and $8 \mathrm{~K}$. It is possible that the functional form assumed for $C(T)$ by Gaiser et al. [24] is not the right shape to represent this system.

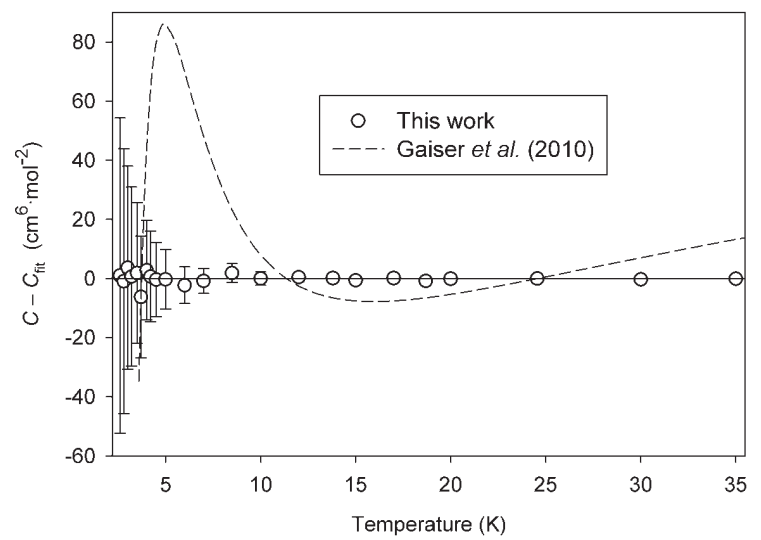

Fig. 3. Deviation of PIMC results and of low-temperature $C(T)$ data of Gaiser et al. [24] from fitted $C(T)$ as given by Eq. (22).

\subsection{Comparison With Experiment for $\gamma_{a}$}

In this section, we compare our results for $R T \gamma_{a}$ with measurements spanning the temperature range $3 \mathrm{~K}$ to $423 \mathrm{~K}$. In this range, $R T \gamma_{\mathrm{a}}$ has a strong temperature dependence; it increases from approximately $-13000 \mathrm{~cm}^{6} \cdot \mathrm{mol}^{-2}$ at $3 \mathrm{~K}$ to approximately $900 \mathrm{~cm}^{6} \cdot \mathrm{mol}^{-2}$ near $14 \mathrm{~K}$ and then decreases to $-9 \mathrm{~cm}^{6} \cdot \mathrm{mol}^{-2}$ at $423 \mathrm{~K}$. Throughout most of this range, the uncertainty of our PIMC values of $R T \gamma_{\mathrm{a}}$ is on the order of $0.5 \%$ to $3 \%$. Because of the wide range and precision required, we do not compare our results with measurements on a conventional graph. Instead, we have plotted the quantity $10^{-3}(T / \mathrm{K})^{1.5} \times R T \gamma_{\mathrm{a}}$, where the exponent 1.5 was chosen so that the range of the product $10^{-3}(T / \mathrm{K})^{1.5} \times R T \gamma_{\mathrm{a}}$ in the interval from $3 \mathrm{~K}$ to $423 \mathrm{~K}$ is much smaller than the range of $R T \gamma_{\mathrm{a}}$ (see Fig. 4).

We examined the speed-of-sound data for ${ }^{4} \mathrm{He}$ published in archival journals and found three publications from which we could determine accurate values of $\gamma_{a}$ [25-27]. Remarkably, the most recent of these publications is 35 years old. Thus, these studies did not benefit from the dramatic reduction in the uncertainty of speed-of-sound measurements that acoustic thermometry has achieved during the past 20 years [3, 28-30].

Gammon [25] measured the speed of sound of ${ }^{4} \mathrm{He}$ on 14 isotherms spanning the temperature range $98 \mathrm{~K}$ to $423 \mathrm{~K}$ at intervals of $25 \mathrm{~K}$ and spanning the pressure range $10 \mathrm{~atm}$ to $150 \mathrm{~atm}$ at intervals of $10 \mathrm{~atm}$ 


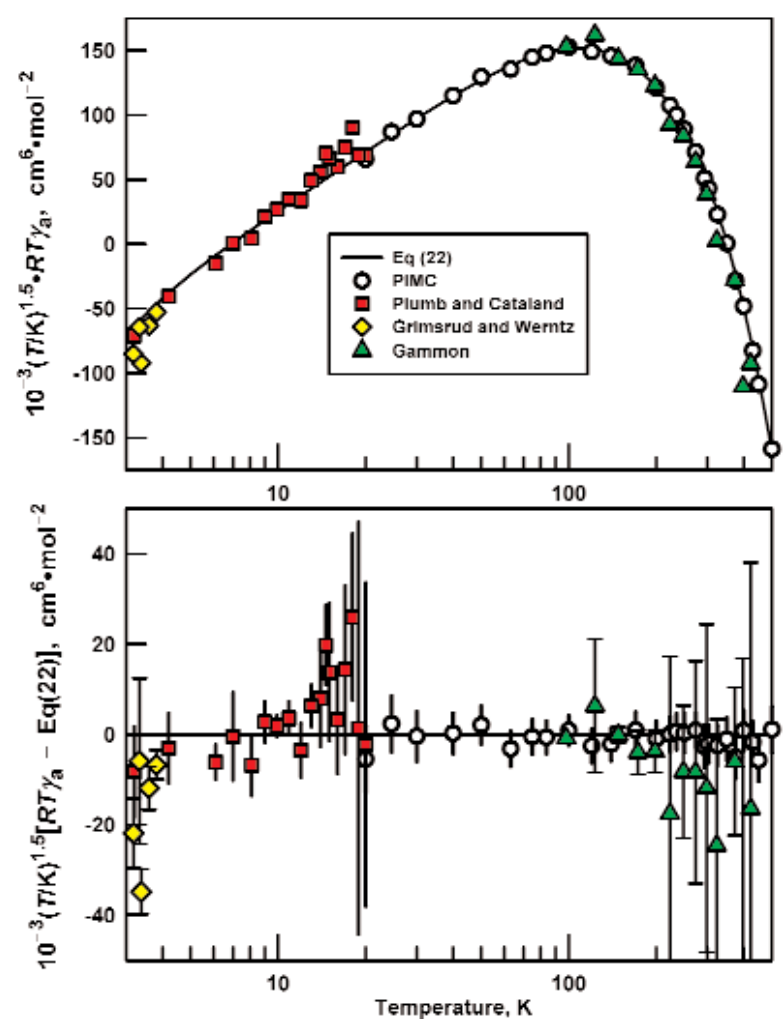

Fig. 4. Comparison with experimental results of third acoustic virial coefficients $\gamma_{\mathrm{a}}$ for ${ }^{4} \mathrm{He}$ calculated in this work both directly with pathintegral Monte Carlo (PIMC), and with $\gamma_{\mathrm{a}}$ calculated with the use of derivatives of the $C(T)$ correlation Eq. (22).

$(1 \mathrm{~atm}=0.101325 \mathrm{MPa})$. Gammon correlated his data using a classical model He-He pair potential. From his correlation, he identified the isotherm at $348 \mathrm{~K}$ and 10 other isolated measurements as outliers. We ignored Gammon's isolated outliers and fit the remaining data on each isotherm, including $348 \mathrm{~K}$, with the function

$$
Z_{\mathrm{a}}-\frac{\beta_{\mathrm{a}} p}{R T_{\mathrm{lab}}}=\frac{T}{T_{\mathrm{lab}}}+\frac{\gamma_{\mathrm{a}} p^{2}}{R T_{\mathrm{lab}}}+\frac{\delta_{\mathrm{a}} p^{3}}{R T_{\mathrm{lab}}}+\frac{\varepsilon_{\mathrm{a}} p^{4}}{R T_{\mathrm{lab}}}
$$

In Eq. (24), we define $Z_{\mathrm{a}} \equiv M u^{2} /\left(\gamma_{0} R T_{\text {lab }}\right)$ as the square of the speed of sound divided by its ideal-gas value, and we calculated it from Gammon's tabulated values of $u^{2}$. The values of $Z_{\mathrm{a}}$ have the narrow range 1 to 1.38 ; therefore, we weighted every data point on each isotherm equally. We took $\beta_{\mathrm{a}}$ from Cencek et al. [9], and we fitted the parameters $T / T_{\mathrm{lab}}, \gamma_{\mathrm{a}} /\left(R T_{\mathrm{lab}}\right)$, and, when they are statistically unequal to zero, $\delta_{\mathrm{a}} /\left(R T_{\text {lab }}\right)$ and $\varepsilon_{\mathrm{a}} /\left(R T_{\mathrm{lab}}\right)$. [Here, $T$ is the thermodynamic tempera- ture of each isotherm and $T_{\text {lab }}$ is the temperature reported by Gammon after adjustment for the changes in the internationally accepted temperature scale. The parameter $T / T_{\text {lab }}$ also accounts for possible changes in $M / \gamma_{0}$ that would occur if there were impurities in the helium.] Seven of Gammon's 14 isotherms (98 K, $148 \mathrm{~K}, 173 \mathrm{~K}, 198 \mathrm{~K}, 248 \mathrm{~K}, 273 \mathrm{~K}$, and $373 \mathrm{~K}$ ) were very well behaved; that is, the deviations from a fit with an appropriate number of terms had no obvious pressure dependence. The standard deviation of $Z_{\mathrm{a}}-\beta_{\mathrm{a}} p /\left(R T_{\text {lab }}\right)$ from the fits, averaged over these isotherms, was 0.000014 . For the remaining isotherms, the deviations from the fits are neither random nor have single, outlying points. Therefore, we are unable to rigorously estimate the uncertainties of $\gamma_{\mathrm{a}} / R T$. [We confirmed Gammon's identification of the $348 \mathrm{~K}$ isotherm as anomalous because a satisfactory fit required the term $\delta_{\mathrm{a}} p^{3} /\left(R T_{\mathrm{lab}}\right)$ even though the adjacent isotherms ( $323 \mathrm{~K}$ and $373 \mathrm{~K}$ ) did not.] For the 13 isotherms (excluding $348 \mathrm{~K}$ ), we estimated the standard uncertainty $u\left(\gamma_{\mathrm{a}} / R T\right)$ by multiplying the result of the fitting routine by $\left(\chi^{2} / N\right)^{1 / 2}$, where $N$ is the number of degrees of freedom and $\chi^{2}$ is the sum of the squares of the deviations of the data from the function fitted to it. All of the tabulated (Table 4) uncertainties reflect multiplication of $u\left(R T \gamma_{\mathrm{a}}\right)$ by an additional factor of two to approximate a $95 \%$ confidence limit.

The values of $R T \gamma_{\mathrm{a}}$ and their expanded uncertainties $U\left(R T \gamma_{\mathrm{a}}\right)$ resulting from fitting Gammon's data are displayed in Fig. 4 and Table 4. Our calculations and Gammon's data agree within combined uncertainties, even though Gammon's values of $R T \gamma_{\mathrm{a}}$ are more negative than our calculated values near the upper end of his temperature range. The uncertainties from fitting acoustic data will be underestimated if they do not account for the bias introduced by truncating the virial expansion. We crudely estimate the effect of truncation by comparing the 2nd and 4th columns in Table 4 . For Gammon's isotherms at $98 \mathrm{~K}, 123 \mathrm{~K}, 148 \mathrm{~K}$, and $173 \mathrm{~K}$, Table 4 compares $R T \gamma_{\mathrm{a}}$ obtained with and without the term $\varepsilon_{\mathrm{a}} p^{4} /\left(R T_{\text {lab }}\right)$ in Eq. (24). The two values of $R T \gamma_{\mathrm{a}}$ agree within combined uncertainties. Table 4 also compares values of $R T \gamma_{\mathrm{a}}$ obtained with and without the term $\delta_{\mathrm{a}} p^{3} /\left(R T_{\text {lab }}\right)$ on the isotherms $198 \mathrm{~K}$ through $298 \mathrm{~K}$. Except at $298 \mathrm{~K}$, the two values of $R T \gamma_{\mathrm{a}}$ on each isotherm are mutually consistent. Above $298 \mathrm{~K}, \delta_{\mathrm{a}}$ was zero, within its uncertainty; however, the values of $R T \gamma_{\mathrm{a}}$ could be influenced by contributions from $\delta_{\mathrm{a}}$. We verified that the uncertainties of the values of a $\beta_{\mathrm{a}}$ from Cencek et al. [9] did not contribute significantly to the uncertainty of $\gamma_{a}$. 
Table 4. Third acoustic virial coefficient for ${ }^{4} \mathrm{He}$ and its expanded $(k=2)$ uncertainty from the data of Gammon [25], excluding the isotherm at $348 \mathrm{~K}$. Asterisks indicate fits including additional terms, as discussed in the text

\begin{tabular}{ccccc}
\hline$T$ & $\begin{array}{c}R T \gamma_{\mathrm{a}} \\
\mathrm{K}\end{array}$ & $\begin{array}{c}U\left(R T \mathrm{\gamma}_{\mathrm{a}}\right) \\
\mathrm{cm}^{6} \cdot \mathrm{mol}^{-2}\end{array}$ & $\begin{array}{c}\left(R T \gamma_{\mathrm{a}}\right)^{*} \cdot \mathrm{mol}^{-2} \\
\mathrm{~cm}^{6} \cdot \mathrm{mol}^{-2}\end{array}$ & $\begin{array}{r}{\left[U\left(R T \gamma_{\mathrm{a}}\right)\right]^{*}} \\
\mathrm{~cm}^{6} \cdot \mathrm{mol}^{-2}\end{array}$ \\
\hline 98.15 & $153.2^{\mathrm{a}}$ & 1.0 & $155.1^{\mathrm{b}}$ & 2.5 \\
123.16 & $106.0^{\mathrm{a}, \mathrm{c}}$ & 6.3 & $115.3^{\mathrm{b}}$ & 10.5 \\
148.16 & $77.5^{\mathrm{a}}$ & 1.0 & $80.7^{\mathrm{b}}$ & 2.5 \\
173.16 & $57.8^{\mathrm{a}}$ & 2.1 & $57.9^{\mathrm{b}}$ & 8.0 \\
198.16 & 32.4 & 4.1 & $42.8^{\mathrm{a}}$ & 1.6 \\
223.16 & 22.4 & 2.3 & $27.0^{\mathrm{a}}$ & 10.2 \\
248.16 & 17.1 & 0.9 & $20.9^{\mathrm{a}}$ & 3.7 \\
273.15 & 8.2 & 1.3 & $13.8^{\mathrm{a}}$ & 5.3 \\
298.15 & 7.3 & 6.9 & $21.2^{\mathrm{a}}$ & 27.4 \\
323.13 & 0.4 & 4.7 & & \\
373.13 & -3.8 & 11.9 & & \\
398.15 & -13.5 & 40.5 & & \\
423.15 & -10.4 & 26.0 & & \\
\hline
\end{tabular}

${ }^{\text {a }}$ Fit included the term $\delta_{\mathrm{a}} p^{3} /\left(R T_{\mathrm{lab}}\right)$.

${ }^{\mathrm{b}}$ Fit included the terms $\delta_{\mathrm{a}} p^{3} /\left(R T_{\mathrm{lab}}\right)$ and $\varepsilon_{\mathrm{a}} p^{4} /\left(R T_{\mathrm{lab}}\right)$.

${ }^{\mathrm{c}}$ Not recommended.

Plumb and Cataland [27] measured the speed of sound in ${ }^{4} \mathrm{He}$ on 21 isotherms from $2.3 \mathrm{~K}$ to $20 \mathrm{~K}$ for the purpose of determining the thermodynamic temperature $T$. As is often done in acoustic thermometry, Plumb and Cataland deliberately restricted their data to low densities; therefore, they were unable to determine meaningful values of $\gamma_{\mathrm{a}}$. Because we have $\beta_{\mathrm{a}}$ from Cencek et al. [9], we were able to determine values of $\gamma_{\mathrm{a}}$ on all of the isotherms except those at $5 \mathrm{~K}$, $2.8 \mathrm{~K}$, and $2.3 \mathrm{~K}$. For each isotherm, the values of $\delta_{\mathrm{a}}$ and $\varepsilon_{\mathrm{a}}$ in Eq. (24) were set equal to zero, and the data were weighted equally. The results are displayed on Fig. 4 and in Table 5.

Grimsrud and Werntz [26] measured the speed of sound in ${ }^{4} \mathrm{He}$ on eight isotherms from $2.13 \mathrm{~K}$ to $3.816 \mathrm{~K}$. They fitted their data by, in effect, adjusting $T / T_{\text {lab }}, \beta_{\mathrm{a}}$, and $\gamma_{\mathrm{a}}$. When analyzing their data, we weighted each measurement using the uncertainties that they tabulated. Because we fixed values of $\beta_{\mathrm{a}}$ from Cencek et al. [9], we were able to determine values of $\gamma_{\mathrm{a}}$ with roughly $1 / 5$ the uncertainty achieved by Grimsrud and Werntz. These values are also shown in Table 5 and on Fig. 4. The values of $R T \gamma_{a}$ from Grimsrud and Werntz are systematically more negative than our calculated values, particularly at the lowest temperatures where, for reasons discussed in the next section, the measurements may be more accurate than our calculations.
Table 5. Third acoustic virial coefficient for ${ }^{4} \mathrm{He}$ and its expanded $(k=2)$ uncertainty from the data of Grimsrud and Werntz [26] and from Plumb and Cataland [27]

\begin{tabular}{|c|c|c|}
\hline $\begin{array}{l}T \\
\mathrm{~K}\end{array}$ & $\begin{array}{c}R T \gamma_{\mathrm{a}} \\
\mathrm{cm}^{6} \cdot \mathrm{mol}^{-2}\end{array}$ & $\begin{array}{c}U\left(R T \gamma_{\mathrm{a}}\right) \\
\mathrm{cm}^{6} \cdot \mathrm{mol}^{-2}\end{array}$ \\
\hline \multicolumn{3}{|c|}{ Grimsrud and Werntz [26] } \\
\hline 3.816 & -7100 & 440 \\
\hline 3.595 & -9300 & 700 \\
\hline 3.379 & -14800 & 790 \\
\hline 3.337 & -10600 & 3000 \\
\hline 3.182 & -15000 & 1400 \\
\hline 2.978 & -16400 & 1300 \\
\hline 2.671 & -19500 & 4300 \\
\hline 2.13 & -46000 & 7500 \\
\hline \multicolumn{3}{|c|}{ Plumb and Cataland [27] } \\
\hline 20 & 770 & 400 \\
\hline 18.9 & 840 & 560 \\
\hline 18 & 1180 & 240 \\
\hline 17 & 1070 & 270 \\
\hline 16 & 940 & 190 \\
\hline 15 & 1140 & 260 \\
\hline 14.6 & 1260 & 160 \\
\hline 14 & 1070 & 210 \\
\hline 13 & 1050 & 100 \\
\hline 12 & 820 & 140 \\
\hline 10.9 & 970 & 110 \\
\hline 9.9 & 854 & 75 \\
\hline 9 & 780 & 170 \\
\hline 8.1 & 190 & 300 \\
\hline 7 & 30 & 530 \\
\hline 6.1 & -990 & 260 \\
\hline 4.2 & -4740 & 910 \\
\hline 3.2 & -12400 & 1800 \\
\hline 5 & 1400 & 5100 \\
\hline
\end{tabular}

In the narrow region of overlap, the data of Grimsrud and Werntz [26] are consistent with the data of Plumb and Cataland [27]. As the temperature decreases, both sets of data tend towards values of $R T \gamma_{\mathrm{a}}$ smaller than those derived from our Eq. (22).

Additional values for $\gamma_{\mathrm{a}}$ derived from acoustic experiments between $2.3 \mathrm{~K}$ and $34 \mathrm{~K}$ were reported in a conference proceeding by Plumb [31]. Unfortunately, the actual measured data were never reported, so we were not able to apply new high-accuracy values of $\beta_{\text {a }}$ [9] to obtain values of $\gamma_{a}$ consistent with the best current knowledge, as we did for Refs. [25-27]. We therefore do not show the data from Ref. [31] in Fig. 4, 
but we note that the reported values of $\gamma_{\mathrm{a}}$ are generally consistent with our calculated values within the reported uncertainties of Ref. [31]. Our calculated values are also consistent within mutual uncertainties with a recent experimental result for $\gamma_{\mathrm{a}}$ at $273.16 \mathrm{~K}$ by Gavioso et al. [32].

\subsection{Results for ${ }^{3} \mathrm{He}$}

While the primary focus of this work was on the common isotope ${ }^{4} \mathrm{He}$, the same methods can be used for ${ }^{3} \mathrm{He}$, which is of interest for cryogenic temperature metrology. Table 6 presents values of $C(T)$ for ${ }^{3} \mathrm{He}$, along with their expanded uncertainties. More extensive

Table 6. Third virial coefficients $C(T)$ for ${ }^{3} \mathrm{He}$ calculated in this work and our estimates (see Sec. 4.3) of their expanded $(k=2)$ uncertainties $U(C)$

\begin{tabular}{|c|c|c|}
\hline$T$ & $C$ & $U(C)$ \\
\hline K & $\mathrm{cm}^{6} \cdot \mathrm{mol}^{-2}$ & $\mathrm{~cm}^{6} \cdot \mathrm{mol}^{-2}$ \\
\hline 2.6 & 1343. & 62. \\
\hline 2.8 & 1436. & 46. \\
\hline 3 & 1464. & 41. \\
\hline 3.2 & 1462. & 36. \\
\hline 3.5 & 1418. & 29. \\
\hline 3.7 & 1366. & 27. \\
\hline 4.0 & 1298. & 22. \\
\hline 4.2 & 1257. & 19. \\
\hline 4.5 & 1175 & 16. \\
\hline 5 & 1071. & 12. \\
\hline 6 & 895.0 & 9.1 \\
\hline 7 & 775.6 & 6.6 \\
\hline 8.5 & 644.9 & 4.4 \\
\hline 10 & 553.7 & 3.4 \\
\hline 12 & 475.3 & 2.4 \\
\hline 13.8033 & 426.5 & 1.7 \\
\hline 15 & 401.8 & 1.6 \\
\hline 17 & 367.6 & 1.3 \\
\hline 18.689 & 346.9 & 1.0 \\
\hline 20 & 333.53 & 0.87 \\
\hline 24.5561 & 296.76 & 0.67 \\
\hline 30 & 268.91 & 0.52 \\
\hline 35 & 251.22 & 0.41 \\
\hline 50 & 218.56 & 0.26 \\
\hline 100 & 170.63 & 0.13 \\
\hline 150 & 146.71 & 0.10 \\
\hline 200 & 130.762 & 0.081 \\
\hline 273.16 & 114.365 & 0.068 \\
\hline 300 & 109.627 & 0.065 \\
\hline 400 & 95.707 & 0.058 \\
\hline 500 & 85.563 & 0.054 \\
\hline 750 & 68.758 & 0.049 \\
\hline 1000 & 58.168 & 0.047 \\
\hline 1500 & 45.083 & 0.045 \\
\hline 2000 & 37.130 & 0.044 \\
\hline
\end{tabular}

discussion of the ${ }^{3} \mathrm{He}$ calculations at low temperatures, along with comparison with the limited experimental data, is given in Ref. [12]. We note that, in addition to the data sources below $10 \mathrm{~K}$ examined in Ref. [12], values of $C(T)$ for ${ }^{3} \mathrm{He}$ between $14 \mathrm{~K}$ and $60 \mathrm{~K}$ were reported by Karnus [20]; these seem to be systematically high below about $30 \mathrm{~K}$, similar to the data for ${ }^{4} \mathrm{He}$ from the same study shown in Fig. 2.

For the same reason discussed for ${ }^{4} \mathrm{He}$ in Sec. 4.1, the values in Table 6 differ slightly from those reported in Ref. [12], and the new values in Table 6 should be preferred.

\subsection{Accuracy of Semiclassical Calculations}

In Ref. [4], we assessed the accuracy of a first-order semiclassical calculation of $C(T)$, concluding that the semiclassical results were adequate (in the sense of reproducing the fully quantum $C(T)$ from PIMC calculations within their expanded uncertainties at the $k=2$ level) at temperatures above about $120 \mathrm{~K}$. That conclusion can be reassessed in light of the reduced uncertainties achieved in this work. The semiclassical $C(T)$ deviates from our new PIMC results by more than the expanded uncertainty of our new results at temperatures below about $280 \mathrm{~K}$. For example, at $273.16 \mathrm{~K}$, the semiclassical calculation yields $112.939 \mathrm{~cm}^{6} \cdot \mathrm{mol}^{-2}$, which exceeds the PIMC value by slightly more than the expanded uncertainty given in Table 1.

\section{Discussion}

The availability of new, state-of-the-art pair and three-body potentials has allowed us to calculate $C(T)$ for helium with uncertainties approximately one-fourth that of our previous work [4]. In addition, we have extended the temperature range of our results, which previously had a lower bound of $24.5661 \mathrm{~K}$, to $2.6 \mathrm{~K}$. We also calculated $C(T)$ for the ${ }^{3} \mathrm{He}$ isotope. The incorporation of exchange effects (non-Boltzmann statistics) was necessary to achieve accurate results for both isotopes below about $7 \mathrm{~K}$.

Within the temperature range covered in our previous work [4], our new results given in Table 1 are consistent with our previous results. The present $C(T)$ are somewhat higher than those calculated previously, typically by an amount near one-half of the expanded $(k=2)$ uncertainties of the results in Ref. [4]. This change is primarily due to the more accurate three-body potential used here [11], and is consistent with a few preliminary calculations using the potential of Ref. [11] that were reported in Ref. [4]. 
We extended the PIMC method to calculate the third acoustic virial coefficient $\gamma_{\mathrm{a}}$; our results are consistent with values of $\gamma_{a}$ obtained from experimental acoustic data, and have smaller uncertainties above $18 \mathrm{~K}$. However, the statistical uncertainty in the PIMC calculation of $\gamma_{\mathrm{a}}$ becomes quite large at lower temperatures. We believe more reliable values are obtained by differentiating Eq. (22) to obtain the temperature derivatives of $C$ and then using those values in Eq. (11). Values derived in that way are consistent with experimental results for $\gamma_{\mathrm{a}}$ in the entire range of our $C(T)$ correlation, with the possible exception of the lowest temperatures (below $4 \mathrm{~K}$ ), where the experimental data lie slightly below our results. One might expect this approach to be less reliable at the low end of the temperature range of Eq. (22), where the uncertainty in the points to which the $C(T)$ function was fitted is larger and $\mathrm{d} C / \mathrm{d} T$ and $\mathrm{d}^{2} C / \mathrm{d} T^{2}$ derived from Eq. (22) would have relatively large uncertainties.

At low temperatures, the uncertainty of the present results for $C(T)$ is dominated by the statistical uncertainty of the PIMC integration. This could, of course, be improved somewhat simply by applying more computing resources. Above about $35 \mathrm{~K}$, the uncertainty from the three-body potential becomes the largest contribution. Therefore, for metrology near room temperature, further improvement in the three-body potential would be desirable; a reduction in by a factor of two in the uncertainty of the three-body potential would produce a reduction by almost that factor in the uncertainty of $C(T)$ at room temperature.

The present results could also be extended to lower temperatures at the expense of more computer time. This could have some application in primary thermometry at these temperatures.

One could perform similar calculations for the "cross" third virial coefficients that characterize isotopic mixtures; these would be $C_{334}(T)$, representing interactions among two ${ }^{3} \mathrm{He}$ atoms and one ${ }^{4} \mathrm{He}$ atom, and the similarly defined $C_{344}(T)$. Because the natural abundance of ${ }^{3} \mathrm{He}$ is tiny, contributions from these coefficients would be insignificant for experiments with naturally occurring helium. We are not aware of any situation in metrology where these mixture coefficients would be useful, but, if needed, the extension of the methods used here to the mixture coefficients would be straightforward.

Another quantity of interest is the fourth virial coefficient $D(T)$, whose calculation would be a straightforward extension of our methods. With the reduction in the uncertainty of $C(T)$ achieved in the present work,
$D(T)$ will become the largest uncertainty in some situations in metrology [7]. In principle, calculating $D(T)$ requires not only pair and three-body potentials but also the nonadditive four-body potential. Such a potential would be difficult to develop, but the relatively small size of the three-body effects in helium suggests that one might be able to assume the nonadditive four-body effects were negligible. It should be possible to test that assumption by performing a few high-level ab initio calculations for simple assemblies of four helium atoms (such as tetrahedrons or squares). The calculation for $D(T)$ would require major computing resources because of the increased dimensionality of the integral, but such a calculation may at least be feasible near room temperature where the number of beads in the ring polymers in the PIMC procedure would be relatively small.

\section{Acknowledgments}

We thank K. Szalewicz and J. B. Mehl for providing information (in some cases prior to publication) on the helium pair potential and properties calculated from it, C. Gaiser for advice on helium $C(T)$ data at low temperatures, K. R. Shaul and D. A. Kofke for performing classical calculations that helped us discover a small error in our previous work, and E. W. Lemmon for fitting Eq. (22). The path-integral Monte Carlo calculations were performed on the computing cluster KORE at Fondazione Bruno Kessler.

\section{References}

[1] J. W. Schmidt, R. M. Gavioso, E. F. May, and M. R. Moldover, Polarizability of Helium and Gas Metrology, Phys. Rev. Lett. 98, 254504 (2007).

[2] B. Fellmuth, Ch. Gaiser, and J. Fischer, Determination of the Boltzmann Constant-Status and Prospects, Meas. Sci. Technol. 17, R145 (2006).

[3] L. Pitre, M. R. Moldover, and W. L. Tew, Acoustic thermometry: new results from $273 \mathrm{~K}$ to $77 \mathrm{~K}$ and progress towards $4 \mathrm{~K}$, Metrologia 43, 142 (2006).

[4] G. Garberoglio and A. H. Harvey, First-Principles Calculation of the Third Virial Coefficient of Helium, J. Res. Natl. Inst. Stand. Technol. 114, 249 (2009).

[5] J. J. Hurly and J. B. Mehl, ${ }^{4}$ He Thermophysical Properties: New Ab Initio Calculations, J. Res. Natl. Inst. Stand. Technol. 112, 75 (2007).

[6] W. Cencek, M. Jeziorska, O. Akin-Ojo, and K. Szalewicz, Three-Body Contribution to the Helium Interaction Potential, J. Phys. Chem. A 111, 11311 (2007).

[7] M. R. Moldover and M. O. McLinden, Using Ab Initio "Data" to Accurately Determine the Fourth Density Virial Coefficient of Helium, J. Chem. Thermodyn. 42, 1193 (2010). 
[8] M. Przybytek, W. Cencek, J. Komasa, G. Lach, B. Jeziorski, and K. Szalewicz, Relativistic and Quantum Electrodynamics Effects in the Helium Pair Potential, Phys. Rev. Lett. 104, 183003 (2010).

[9] W. Cencek, M. Przybytek, J. Komasa, J. B. Mehl, B. Jeziorski, and K. Szalewicz, Effects of adiabatic, relativistic, and quantum electrodynamics interactions in helium dimer on thermophysical properties of helium, J. Chem. Phys., to be submitted (2011).

[10] M. Jeziorska, W. Cencek, K. Patkowski, B. Jeziorski, and K. Szalewicz, Pair potential for helium from symmetryadapted perturbation theory calculations and from supermolecular data, J. Chem. Phys. 127, 124303 (2007).

[11] W. Cencek, K. Patkowski, and K. Szalewicz, Full-configuration-interaction calculation of three-body nonadditive contribution to helium interaction potential, J. Chem. Phys. 131, 064105 (2009).

[12] G. Garberoglio and A. H. Harvey, Path-integral calculation of the third virial coefficient of quantum gases at low temperatures, J. Chem. Phys. 134, 134106 (2011).

[13] G. Garberoglio, Boltzmann bias grand canonical Monte Carlo, J. Chem. Phys. 128, 134109 (2008).

[14] M. Galassi, J. Davies, J. Theiler, B. Gough, G. Jungman, M. Booth, and F. Rossi, GNU Scientific Library Reference Manual, Network Theory, revised 2nd edition (2006). http://www.gnu.org/software/gsl.

[15] K. A. Gillis and M. R. Moldover, Practical Determination of Gas Densities from the Speed of Sound Using Square-Well Potentials, Int. J. Thermophys. 17, 1305 (1996).

[16] M. F. Herman, E. J. Bruskin, and B. J. Berne, On path integral Monte Carlo simulations, J. Chem. Phys. 76, 5150 (1982).

[17] A. L. Blancett, K. R. Hall, and F. B. Canfield, Isotherms for the He-Ar System at $50{ }^{\circ} \mathrm{C}, 0{ }^{\circ} \mathrm{C}$ and $50^{\circ} \mathrm{C}$ up to $700 \mathrm{Atm}$, Physica 47, 75 (1970).

[18] M. O. McLinden and C. Lösch-Will, Apparatus for wide-ranging, high-accuracy fluid $(p, \rho, T)$ measurements based on a compact two-sinker densimeter, J. Chem. Thermodyn. 39, 507 (2007).

[19] D. White, T. Rubin, P. Camky, and H. L. Johnston, The virial coefficients of helium from 20 to $300^{\circ} \mathrm{K}$, J. Phys. Chem. 64, 1607 (1960).

[20] A. I. Karnus, Virial Coefficients and some Thermodynamic Quantities of the Helium Isotopes in Low Temperature Range, Ukr. Fiz. Zh. 21, 1179 (1976).

[21] K. H. Berry, Measurements of the second and third virial coefficients of ${ }^{4} \mathrm{He}$ in the range 2.6-27.1 K, Mol. Phys. 37, 317 (1979).

[22] D. Gugan and G. W. Michel, Measurements of the polarizability and of the second and third virial coefficients of ${ }^{4} \mathrm{He}$ in the range 4.2-27.1 K, Mol. Phys. 39, 783 (1980).

[23] L. V. Karnatsevich, I. V. Bogoyavlenskii, and L. P. Titar, Virial coefficients of helium isotopes at low temperatures, Sov. J. Low Temp. Phys. 14, 1 (1988).

[24] C. Gaiser, B. Fellmuth, and N. Haft, Dielectric-Constant GasThermometry Scale from $2.5 \mathrm{~K}$ to $36 \mathrm{~K}$ Applying ${ }^{3} \mathrm{He},{ }^{4} \mathrm{He}$, and Neon in Different Temperature Ranges, Int. J. Thermophys. 31, 1428 (2010).

[25] B. E. Gammon, The velocity of sound with derived state properties in helium at -175 to $150{ }^{\circ} \mathrm{C}$ with pressure to $150 \mathrm{~atm}, \mathrm{~J}$. Chem. Phys. 64, 2556 (1976).
[26] D. T. Grimsrud and J. H. Werntz, Measurements of the Velocity of Sound in $\mathrm{He}^{3}$ and $\mathrm{He}^{4}$ Gas at Low Temperatures with Implications for the Temperature Scale, Phys. Rev. 157, 181 (1967).

[27] H. Plumb and G. Cataland, Acoustical Thermometer and the National Bureau of Standards Provisional Temperature Scale 2-20 (1965), Metrologia 2, 127 (1966).

[28] A. F. Estrada-Alexanders and J. P. M. Trusler, The speed of sound in gaseous argon at temperatures between $110 \mathrm{~K}$ and $450 \mathrm{~K}$ and at pressures up to $19 \mathrm{MPa}$, J. Chem. Thermodyn. 27, 1075 (1995).

[29] G. Benedetto, R. M. Gavioso, R. Spagnolo, P. Marcarino, and A. Merlone, Acoustic measurements of the thermodynamic temperature between the triple point of mercury and $380 \mathrm{~K}$, Metrologia 41, 74 (2004).

[30] D. C. Ripple, G. F. Strouse, and M. R. Moldover, Acoustic Thermometry Results from $271 \mathrm{~K}$ to $552 \mathrm{~K}$, Int. J. Thermophys. 28, 1789 (2007).

[31] H. H. Plumb, ${ }^{4} \mathrm{He}$ second and third virial coefficients from acoustical isotherms: The Helmholtz-Kirchhoff correction at temperatures below $35 \mathrm{~K}$, in Temperature: Its Measurement and Control in Science and Industry, vol. 5, J. F. Schooley, Ed. (American Institute of Physics, New York, 1982), Part 1, pp. 77-88.

[32] R. M. Gavioso, G. Benedetto, D. Madonna Ripa, P. A. Giuliano Albo, C. Guianvarc'h, A. Merlone, L. Pitre, D. Truong, F. Moro, and R. Cuccaro, Progress in INRiM Experiment for the Determination of the Boltzmann Constant with a QuasiSpherical Resonator, Int. J. Thermophys., in press, doi:10.1007/s10765-011-1032-9.

About the authors: Giovanni Garberoglio is a physicist and a member of the Interdisciplinary Laboratory for Computational Science (LISC), a collaboration between Fondazione Bruno Kessler and the University of Trento, Italy. Michael Moldover is a physicist and a NIST Fellow; he is the Leader of the Fluid Metrology Group in the Temperature, Pressure, and Flow Metrology Division of the NIST Physical Measurement Laboratory. Allan Harvey is a chemical engineer in the Theory and Modeling of Fluids Group in the Thermophysical Properties Division of the NIST Material Measurement Laboratory. The National Institute of Standards and Technology is an agency of the U.S. Department of Commerce. 